\title{
GUMLAULE
}

CUM LAUDE

Revista del Doctorado en Derecho

Facultad de Derecho y Ciencias Sociales y Políticas UNNE

$\mathrm{N}^{\circ} 1$ - Diciembre 2014

Corrientes - Argentina

ISSN 2422-6408 (formato digital)

info@revistacumlaude.com

FECHA DE RECEPCIÓN: 05/03/2014

FECHA DE ACEPTACIÓN: 22/06/2014

\section{LA IGUALDAD JURÍdicA DE LAS PROVINCIAS ARGENTINAS}

\section{Carlos Daniel luque}

UNiVERSIDAd NACIONAL DEL NORDESTE

\section{RESUMEN}

El concepto de provincia en la estructura constitucional argentina tiene, al menos, dos formas de abordarse y diferenciarse. La primera, la de las provincias fundadoras del Estado Federal luego del largo proceso histórico del derecho patrio argentino, pasando por los pactos preexistentes hasta el acuerdo de San Nicolás de los Arroyos, que dará como fruto la convención de Santa fe y la Constitución Nacional de 1853. La segunda forma de arrancar el estudio de las provincias argentinas es tratar de explicar el alcance que tienen los derechos adquiridos ante el Estado Nacional de aquellas que fueron creadas por ley del Congreso Nacional a partir de los territorios nacionales. Pero ante esta situación necesitamos fortalecer la idea de que en toda federación (y la 
Argentina lo sigue siendo formalmente) existe igualdad jurídica de las provincias, aunque para demostrar la existencia de una "no igualdad jurídica total" entre las provincias, el art. 121, in fine, de la Constitución Nacional marca otra excepción al principio de igualdad de las provincias, al señalar que algunas pueden contar con un cupo especial de derechos: "el que expresamente se hayan reservado por pactos especiales al tiempo de su incorporación". Todo esto hubiese tenido un saldo positivo si la reforma de 1994 hubiera igualado los diferentes status examinados para evitar que esta, para algunos inimaginable, postulación que aquí hacemos pueda darse algún día.

\section{Palabras Clave}

Provincias - Constitución Nacional - Constituciones Provinciales - Corte Suprema de Justicia de la Nación

\section{Abstract}

The concept of province in Argentina's constitutional structure has at least two ways to differentiate and addressed. The first, of the founding provinces of the Federal State after the long historical process of Argentine patriotic duty, to pre-existing agreements until settlement of San Nicolas de los Arroyos, that will grow the Santa Fe Convention and the Constitution of 1853 . The second way to start the study of Argentina's provinces is trying to explain the extent they have acquired rights to the National State of those that were created by an Act of Congress as the national territories. However, in this situation we need to strengthen the idea that in any federation (and Argentina it is formally) there is legal equality of the provinces, but to demonstrate the existence of a "no full legal 
equality" between the provinces, the art. 121, in fine, of the Constitution marks another exception to the principle of equality of the provinces, noting that some may have a special quota of rights, "which expressly reserved by special pacts at the time of incorporation." All this had had a positive balance if the reform of 1994 had matched the different status examined, to prevent that this kind of arguments were possible.

\section{KEYWORDS}

States - Federal Constitution - States Constitution - Supreme Court

\section{LAS PROVINCIAS ORIGINARIAS}

El concepto de provincia en la estructura constitucional argentina tiene, al menos, dos formas de abordarse y diferenciarse.

La primera, la de las provincias fundadoras del Estado Federal luego del largo proceso histórico del derecho patrio argentino, pasando por los pactos preexistentes hasta el acuerdo de San Nicolás de los Arroyos, que dará como fruto la convención de Santa fe y la Constitución Nacional de 1853.

La segunda forma de arrancar el estudio de las provincias argentinas es tratar de explicar el alcance que tienen los derechos adquiridos ante el Estado Nacional de aquellas que fueron creadas por ley del Congreso Nacional a partir de los territorios nacionales.

Dejamos muy en claro aquí al principio que:

la garantía del derecho a la existencia integral, indestructibilidad e integridad territorial de las Provincias, es inherente a su calidad de 
Estados a la vez que a su condición de entidades políticas soberanas (provincias originarias mientras lo fueron) y preexistentes a la Constitución Nacional. (Linares Quintana, 1985, p.349)

Se colige de esto que el derecho constitucional argentino reconoce a las provincias originarias el derecho a existir integralmente, pues tal poder ha sido retenido al conformar el Estado Federal, y es un derecho primigenio e inherente, (Preámbulo, arts. $6^{\circ}$ y $13, \mathrm{CN}$ ) similar al derecho a la vida de las personas individuales (Galiana, 2007).

Ahora bien -y aquí formulamos un interrogante como hipótesis de trabajo-, la calidad de unidades indestructibles prevista para las provincias que originariamente conformaron el Estado, ¿tiene el mismo alcance que para lo que fueron creadas por el poder constituido (Congreso), a través o por medio de una ley?

Nos explicamos. Cuando hablamos de alcance de manera alguna hacemos referencia a, por ejemplo, el alcance de la autonomía provincial, que es igual para todas las provincias argentinas y toda otra cuestión que pudiese derivar de nuestra forma republicana de gobierno, aquí veremos cuestiones de estricto corte normativo, que realmente creemos que es lo que diferencia a las provincias originarias de las creadas por los poderes constituidos de la Nación.

Para resolver esta cuestión no sólo daremos una opinión preliminar sobre el tema y fijaremos, al final de este trabajo, nuestra posición, sino que también nos ocuparemos de dejar clara la opinión de la más destacada doctrina constitucional que tuvo, sobre la cuestión, la segunda mitad de este siglo $\mathrm{XX}$. 
Sabido es que las provincias argentinas entre 1810 y 1853-1860 eran o fueron Estados con plenos poderes dentro de una confederación invertebrada e imperfecta (Galiana, 2007).

Lo demuestra Corrientes, por ejemplo, por sus largos y solitarios desencuentros con Buenos Aires, sus guerras y el ejercicio de su soberanía con expedición de pasaportes, relaciones internacionales, papel moneda, guerra y paz, etc. (Galiana, 2007).

Esta plena soberanía ejercida realmente, que concluye con la Constitución de 1853 , les permite a los estados constituyentes conservar los derechos no delegados a la Nación que entonces forman (Galiana, 2007).

Dentro de esta introducción a la cuestión provincia merece una brevísima consideración la Ciudad Autónoma de Buenos Aires:

la que habiendo sido creada (o su estatus) por una convención constituyente no reúne la calidad de provincia Argentina, y así como fue creada puede ser suprimida pero por otra convención constituyente, fijando un requisito de mayor rigor que las provincias nuevas, aquellas que fueron creadas por ley del poder constituido. (Bidart Campos, 1995, p.537)

Algo similar, y más importante aún para nuestra historia constitucional, había ocurrido al cerrarse el ciclo constitucional originario en 1860 con la vuelta de la Provincia de Buenos Aires, como consecuencia de la firma del Pacto de San José de Flores el 11 de noviembre de 1859, a la Confederación Argentina después de haberse escindido de la misma al no aceptar los términos, o alguno de ellos, de la Constitución sancionada en mayo de 1853 . 
Sirva este relato preliminar para responder de manera aproximada a las preguntas que nos formulamos al iniciar la cuestión de las provincias.

\section{LOS TERRITORIOS NACIONALES}

"El antecedente inmediato de las provincias creadas por ley (nuevas provincias) corresponde a los territorios nacionales" (Academia Nacional de la Historia, 2000, p. 511).

Formar un Estado y delimitar el territorio constituían pasos necesarios, para ello el Congreso tiene facultades de fijar los límites provinciales y disponer el uso de la enajenación de las tierras de propiedad nacional (art. 75, inc. 5 y 15, C.N.) (Anderson, 2000).

En 1882 se dividió la enorme Gobernación de la Patagonia en los ahora llamados territorios nacionales, que eran nueve gobernaciones con asiento en: La Pampa (Gral. Acha), Neuquén (Chos Malal), Río Negro (Viedma), Chubut (Madryn), Santa Cruz, Tierra del Fuego (Ushuaia), Chaco (Colonia Resistencia), Formosa (Villa Formosa) y Misiones (Posadas) (Academia Nacional de la Historia, 2000).

Y en cumplimiento del mandato constitucional el Estado dictó la ley 1532 del 16 de octubre del año 1884 tendiente a la organización, administración y gobierno que debían tener dichos territorios nacionales.

No tenían el mismo estatus que las provincias originarias porque carecían de autonomía por lo que quedaron durante mucho tiempo reducidos al papel de simples divisiones administrativas de la Nación. 
Éste era el paso previo para convertirse en provincia: debían alcanzar los 60.000 habitantes pero no era un proceso mecánico, aunque alcanzarán el número mencionado no se convertirían en provincias inmediatamente, ya que era facultad del Congreso de la Nación, que desde luego la ejercitó cuando le fue conveniente (Galiana, 2007).

Estos territorios tenían un poder ejecutivo elegido por el PEN con acuerdo del Senado, secundado por otro funcionario que refrendaba sus actos, una Legislatura local, gobiernos municipales y una justicia propia (Galiana, 2007)

Los territorios nacionales fueron el fruto de las campañas al desierto que se llevaron a cabo en el siglo XIX culminando recién en el siglo XX con la efectiva ocupación del territorio, aunque parte de ellos estaba en manos de los propietarios originarios de la tierra (Galiana, 2007).

\section{LaS NUEVAS PROVINCIAS}

Luego de muchas idas y vueltas entre la provincialización y la territorialización, entre quienes estaban a favor de una nueva provincia y los que se oponían, aparecerán en el escenario del derecho público provincial las mencionadas nuevas provincias.

Entonces la ley 14.037 creó las provincias de Chaco y La Pampa, la ley 14.294 creó la provincia de Misiones, la ley 14.408 provincializó Formosa, Neuquén, Chubut y Río Negro (1953).

Más tarde se crea por decreto-ley 21.178 la provincia de Santa Cruz, en reemplazo de la provincia de la Patagonia (que también había sido creada 
en el paquete de la ley 14.408 y era Santa Cruz con el anexo de Tierra del Fuego) (Sabat, s.f.); concluyendo el ciclo con la última provincia del mapa argentino: Tierra del Fuego, por ley 23.775 de 1990 (Zarini, 1996).

Como se aprecia la provincia de la Patagonia existió al igual que las otras provincias creadas por ley y, luego, de la misma manera desapareció. Esto nos permite cuando menos considerar con seriedad que una provincia creada por ley puede desaparecer del contexto del Estado argentino.

No obstante lo hasta aquí reseñado, no desconocemos que la mayoría de la doctrina concuerda con los mecanismos a los que refiere el art. 13 de la $\mathrm{CN}$, si bien con algunos matices, como pasamos a ver.

4. LA imposibilidad de Eludir EL ART. 13 de LA Constitución NACIONAL

Spota (1995) al desarrollar la cuestión de la autonomía en la estructura constitucional argentina precisa claramente que "la autonomía de las provincias nace del art. 13 de la Constitución Nacional, en consonancia con el art. $3^{\circ}$.

Esas dos normas definen las características de la autonomía en nuestra estructura constitucional.

De allí se deduce que "por imperio exclusivo de la Constitución Nacional, y en su ámbito, solamente las provincias son autónomas" (Spota, 1995, p. 19).

Desde ya que la condición única, sine qua non, es el cumplimiento de la manda del art. 13 de la Constitución Nacional, cuestión que compartimos 
por otra parte porque en este trabajo, como dijimos al iniciarlo, no se está discutiendo la cuestión de la autonomía provincial sino que objetamos el modo de creación de las denominadas nuevas provincias.

Subraya Spota (1995) que:

el tema de la autonomía está referido fundamentalmente a cómo nace y como muere la misma (autonomía); (ese tema sí es el que queremos revisar aquí: cómo nace y muere una provincia, sobre todo a la luz de los antecedentes tan claros que hemos mencionado) y estoy definitivamente convencido que una autonomía en una estructura federal como la nuestra, requiere para nacer y también para extinguirse, del concurso necesario, imprescindible y no eludible, de dos voluntades. La voluntad del todo federal, y la voluntad de la población que va a gozar de esa autonomía. Si no existe el concurso de esas dos voluntades, no nace una autonomía y tampoco puede extinguirse o modificarse la autonomía existente. (p.20)

Coincidimos con la construcción normativa del profesor de Buenos Aires porque dicho desarrollo es óptimo a la luz de la manda de la Constitución Nacional.

\section{La posición de Germán Bidart Campos o la deSigualdad en la} APliCaCión del art. 13 de la Constitución NaCional

De la manera más sintética posible trataremos de graficar en este lugar el pensamiento que sobre este tema nos acerca Bidart Campos.

El gran constitucionalista argentino del siglo $\mathrm{XX}$ comienza afirmando: "Las provincias son preexistentes al estado federal. Pero ¿cuáles 
son anteriores al estado federal? Solamente las catorce que existían a la fecha de ejercerse el poder constituyente originario (1853-1860) y que dieron origen a la federación en esta etapa" (Bidart Campos, 1999-2000, p.656).

Al referirse a la hipótesis del art. 13 (que prevé que, mediante consentimiento del congreso federal y de la legislatura de las provincias interesadas, puede erigirse una provincia en el territorio de otra u otras, o de varias formarse una sola) que estamos exponiendo con sus distintos matices, Bidart Campos no es menos y dice expresamente, al interpretar la norma, que dicha autorización consentida del mencionado artículo es de dudosa aplicación para las provincias preexistentes al estado federal:

Creemos que no [que el art. 13 no se aplica a las provincias originarias] porque las catorce provincias históricamente anteriores no pueden desaparecer, y de un modo más o menos intenso desaparecerían como unidades políticas si se fusionaran con otra, o si dentro de su territorio se formara una nueva. Por ende [insiste el querido maestro], pensamos que la habilitación que en la cláusula citada contiene el art. 13 sólo tiene virtualidad de aplicación respecto de las nuevas provincias creadas con posterioridad a 1853-1860. (Bidart Campos, 1999-2000, p.657)

Con lo cual, ante semejante afirmación, es claro de la simple lectura del texto transcripto, que para Bidart Campos la norma constitucional aludida no se aplica de manera igualitaria a todas las provincias argentinas.

Para terminar con esta posición decimos que también se refiere a la creación de nuevas provincias por el Congreso Federal y en este particular 
dice que las erige o erigirá en igualdad de estatus jurídico y político que las catorce originarias.

Esta última cuestión, repetimos, nunca estuvo aquí en duda. Para nosotros como para la casi unánime doctrina argentina que se ocupa de la cuestión, las provincias nuevas se integran en paridad e igualdad de situación que las demás.

\section{LA OPINIÓN DEL PUBLICISMO PROVINCIAL}

Brevemente no queremos dejar de mencionar el pensamiento que dejó marcado en esta cuestión el publicismo provincial, en este particular encarnado en la Profesora de Mendoza María Castorina de Tarquini.

Tenemos como principio rector que "(...) toda federación importa, para su consolidación, que el ordenamiento jurídico y el gobierno federal sean supremos respecto de los estados miembros (...)" (Castorina De Tarquini, 2007, p. 164).

Pero ante esta situación necesitamos fortalecer la idea de que en toda federación (y la Argentina lo sigue siendo formalmente) existe igualdad jurídica de las provincias, a este respecto Castorina de Tarquini (2007) refiere: "(...) otro presupuesto del mantenimiento de la relación federal es que, respetando las diversidades y peculiaridades de las provincias, se las coloque jurídicamente en situación de igualdad. No cabe reconocer privilegios a favor de unas provincias y en perjuicio de otras" (p. 164).

Y la opinión se amplía "lo expuesto es válido tanto para las

provincias existentes al momento de dictarse la Constitución Nacional, 
como para las que se incorporan con posterioridad" (Castorina de Tarquini, 2007, p.164).

A la afirmación de la profesora mendocina le asiste la razón en cuanto a su parecer sobre este respecto, pero como dijimos antes esto ocurre en la generalidad de la cuestión y no en la problemática particular que estamos tratando, y lo que dice Castorina de Tarquini aquí no se está discutiendo a pesar de que estamos totalmente de acuerdo con ella.

\section{La Cuestión en la CoRte}

Tenemos, en principio, que la Corte Suprema ha reconocido la preexistencia de las provincias históricas a la Nación (Buenos Aires, Córdoba, Catamarca, Santa Fe, San Juan, Entre Ríos, Corrientes, Salta, Mendoza, San Luis, Santiago del Estero, Jujuy, Tucumán y La Rioja) (CSJ Fallos: 1:170).

En cuanto a la igualdad de las provincias dice Sagües (2007) que: la Corte, en "Prov. de la Pampa c. Prov. de Mendoza", aplicó el principio estadounidense de la igualdad política, a las provincias que forman la federación argentina, aunque, ello no importaba su igualdad económica (...) citando a la Corte Suprema norteamericana, nuestro alto tribunal explicó que nunca ha habido igualdad entre los Estados en ese sentido. (p. 455)

Insiste Sagüés (2007) con el tema y se pregunta: “¿están en igualdad de trato las provincias viejas (esto es, las catorce existentes al momento de sancionarse la Constitución de 1853-1860) y las creadas después?”(p.455), 
y la respuesta es que no, que también existen otras salvedades a la regla de igualdad política antes mencionada.

El problema también fue atendido por la Corte para decidir sobre los actos de disposición adoptados por el Gobierno nacional respecto de bienes de un territorio federal antes de que en él fuera erigida una nueva provincia (CSJ Fallos: 310:2527).

En definitiva, la Corte Suprema exigió que esas decisiones, para obligar a una nueva provincia, debían haber contemplado el interés de la misma; por eso declaró no vinculatorio para la provincia de La Pampa el acuerdo celebrado entre la Nación y la Provincia de Mendoza, sobre el río interprovincial Atuel, suscripto cuando La Pampa era sólo territorio nacional (CSJ Fallos: 256:588).

Finalmente y para demostrar la existencia de una "no igualdad jurídica total" entre las provincias, el art. 121, in fine, de la Constitución Nacional marca otra excepción al principio de igualdad de las provincias, al señalar que algunas pueden contar con un cupo especial de derechos: "el que expresamente se hayan reservado por pactos especiales al tiempo de su incorporación"; clara remisión aquí al Pacto de San José de Flores cuya vigencia, según la Corte, no podría ser alterada unilateralmente por la Nación, pues para ello es necesaria la voluntad de la provincia pactante.

Rescatamos otro ejemplo para fortalecer lo que venimos diciendo y para no quedarnos únicamente con el ejemplo de la Patagonia que tratamos allá por el capítulo tercero (recordamos brevemente, una ley del Congreso 
creó la Provincia de la Patagonia y otra la derogó creando, en su lugar, la Provincia de Santa Cruz).

En 1881 a través de una ley nacional por la que se establecieron los límites de la provincia de Corrientes, se separó de la misma a la provincia de Misiones incorporada está a la primera desde 1814. Con el tiempo Misiones adquirió su calidad de provincia como vimos, pero en 1881 Corrientes no prestó el consentimiento requerido por la Constitución Nacional, para el desapoderamiento del que fue objeto (Midón, 1997).

\section{OPINIONES CONCLUSIVAS}

Concluyendo podemos corroborar el dato de que el Congreso puede, en el caso de provincias creadas por ley, extinguirlas o suprimirlas, entendiéndose que si a una provincia fundadora y originaria como Corrientes pudieron extirparle su territorio incumpliendo la manda del art. 13 de la CN más aún se podrá hacer con simples provincias creadas por ley.

La hipótesis formulada resulta cuasi-imaginativa (o no tanto), pero si colocamos el análisis del tema dentro del marco del "realismo jurídico" argentino, es posible que nos pongamos a pensar qué puede llegar a ocurrir, por cuestiones políticas, si al Congreso de la Nación se le ocurre el dictado de una ley que suprima la existencia de una nueva provincia que en su momento fue creada por el mismo instrumento legal.

Más aunque forzando dicho "realismo jurídico", y con el nuestra postura, así como hay leyes que intervienen provincias con causa y sin causa, y teniendo en cuenta la ligereza que demuestran los fundamentos de 
dichos instrumentos legislativos, quienes avalan esas leyes de intervención, de inimaginable factura, tranquilamente podrían y podrán avalar leyes del congreso para suprimir una provincia no fundacional (Galiana, 2007).

Finalizamos relatando que todo este marco teórico trabajado ya ocurrió y sostiene, en gran parte, lo posición sustentada.

Nos referimos a que la supresión de una provincia ya es parte de nuestra historia constitucional, la provincia Patagonia fue suprimida por un decreto-ley (Galiana, 2007), por lo tanto entendemos que nuestra hipótesis, aunque pueda no gustar o parecer anti-práctica, es totalmente realizable.

La solución más eficaz pero a la vez también la más legitima hubiese sido igualarlas constitucionalmente en la reforma de 1994, a través de una simple frase en forma de artículo en nuestra ley mayor para evitar que esta postulación que aquí desarrollamos pueda, en algún momento, hacerse realidad.

\section{REFERENCIAS BIBLIOGRÁFICAS}

Academia Nacional de Historia. (2000). Nueva historia de la Nación Argentina (tomo 4). Buenos Aires: Planeta.

Anderson, B. (2000). Comunidades Imaginadas. Buenos Aires: FCE.

Bidart Campos, G. J. (1995). Tratado elemental de Derecho Constitucional Argentino (tomo 4). Buenos Aires: Ediar.

Bidart Campos, G. J. (1999-2000). Tratado elemental de Derecho Constitucional Argentino (tomo 1-A). Buenos Aires: Ediar. 
Castorina de Tarquini, M. C. (2007). Derecho Público Provincial y municipal (María Gabriela Ábalos act.). Buenos Aires: La Ley.

Corte Suprema de Justicia de la Nación (s.f.). Colección de fallos, 1:170, $310: 2527,256: 588$.

Galiana, E. E. (2007). Orden y Progreso - Provincias Fundadoras y Nuevas Provincias. Corrientes: Moglia Ediciones.

Linares Quintana, S. (1985). Tratado de la Ciencia del Derecho Constitucional (tomo 7). Buenos Aires: Plus Ultra.

Midón, M. A. R. (1997). Manual de Derecho Constitucional Argentino. Buenos Aires: Plus.

Sabat, J. M. (s.f.) Los Territorios y su administración militar en la legislación argentina. ED, T99-929.

Sagues, N. P. (2007). Manual de Derecho Constitucional. Buenos Aires: Astrea.

Spota, A. A. (1995). Las Autonomías de los municipios provinciales en la reforma constitucional de 1994. Separata de Anales de la Academia de Ciencias Morales y Políticas.

Ensayo sobre el artículo 123 de la Constitución Nacional y la autonomía de los municipios. (2001). Boletín Informativo de la Asociación Argentina de Derecho Constitucional, 177.

Zarini, J. H. (1996). Constitución Argentina Comentada y Concordada. Buenos Aires: Astrea. 


\section{Curriculum vitae}

Abogado por la Universidad Nacional del Nordeste. Profesor de Derecho Constitucional (JTP) en la Catedra "A" de Derecho Constitucional de la Facultad de Derecho y Ciencias Sociales y Políticas de la Universidad del Nordeste. Miembro de la nómina actual de Investigadores consolidados de la Facultad de Derecho, Ciencias Sociales y Políticas de la Universidad Nacional del Nordeste. Integrante del Proyecto de Investigación de la Secretaria de Ciencia y Técnica de la Universidad Nacional del Nordeste: P.I. G008-11 “Conciencia Jurídica Material. Legislación Nacional e Internacional. Implicancia de los tratados internacionales en el fallo Arancibia Clavel" (en ejecución). Miembro Titular de la Asociación Argentina de Derecho Constitucional. Correo electrónico: carlosdluque@hotmail.com 